\title{
ULTRASONOGRAPHIC EXAMINATION OF SOME VESSELS IN DOGS AND THE CHARACTERISTICS OF BLOOD FLOW IN THESE VESSELS
}

\author{
Figurová, M., Kulinová, V. \\ Small Animal Clinic \\ University of Veterinary Medicine and Pharmacy, Komenského 73, 04181 Košice \\ Slovakia \\ maria.figurova@uvlf.sk
}

\begin{abstract}
The examination by Doppler ultrasonography provides haemodynamic information about blood flow velocity in a respective vessel. It specifies high- and lowresistance flow patterns. The aim of our study was to record the flow in a. carotis communis, a. femoralis and aa. renales in $\mathbf{1 6}$ adult clinically healthy dogs of small and medium size; characterize the types of vessels and also determine the pulsatility index (PI) and the resistive index (RI) of these vessels. The a. femoralis is a high-resistance vessel with a pronounced three-peak waveform. The aa. renales gives a typical picture of a low-resistance flow pattern. The characteristics of $a$. carotis communis involves different images of its branches $a$. carotis inter$n a$ and $a$. carotis externa. In the investigated groups we observed a medium degree of pulsatility (atypical highresistance flow pattern with an absence of reverse flow). The mean measured values of indices for a. carotis communis were: left side PI 1.824 and RI 0.742; right side PI 1.891 and RI 0.746, and for aa. renales: PI $1.366 \pm 0.04$ and RI $0.684 \pm 0.05$.
\end{abstract}

Key words: Duplex ultrasonography; pulsatility index PI; resistive index RI

\section{INTRODUCTION}

Doppler ultrasonography is a precise non-invasive method used for the examination of blood flow, its direction, velocity and character in a blood vessel. It involves the physical interaction between ultrasound and the flowing blood (Fig. 1). The Doppler effect is a result of an apparent shift in frequency of the sound reflected from the moving target particles, i.e. the blood cells. The frequency of the reflected sound differs from the original frequency, a phenomenon referred to as a Doppler shift. We recognise four ways of Doppler imaging based on various physical principles of image production: pulsed wave (PW), continuous wave $(\mathrm{CW})$, colour Doppler and power Doppler.

Doppler imaging helps to distinguish vessels from nonvascular structures, such as dilated biliary duct or renal diverticulum. Its practical use involves pathological imaging of vascular lumen, e.g. in case of a suspected thrombus 


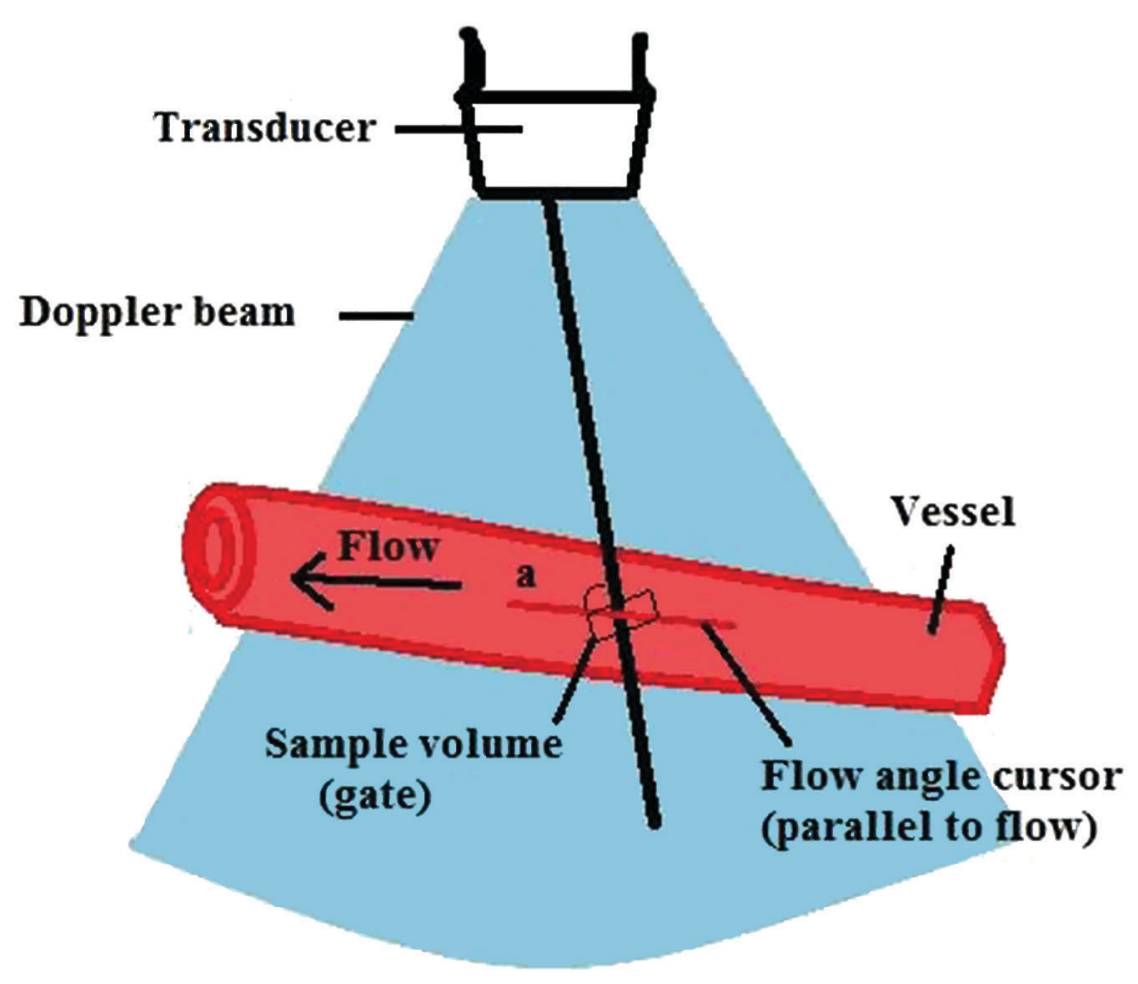

Fig. 1. Doppler principle Source: Mattoon and Nyland [8]

obturation, during localisation of pathological interconnection of vessels (porto system shunt) or examination of blood flow in the splanchnic region or detection of arteriovenous (AV) abnormalities and tumours. It is irreplaceable in cardiology for the examination of insufficiencies of valves, stenosis or septal defects [8].

\section{Sonographic imaging of vessels in Real-time B-mode}

Abdominal vessels have tubular structure in the longitudinal plane with well-defined walls. The walls are parallel, hyperechogenic, appearing as thin smooth lines. In the cross-section, the non-compressed vessels appear as circular or oval. The lumen is anechogenic due to the presence of blood, which does not produce an echo by itself (Fig. 2) [12]. At low velocity of flow and sufficient vessel diameter, the aggregated red blood cells can be observed as moving echoes. The two-dimensional real-time image is suitable for the detection of abnormally localised vessels (shunts), measurement of diameter (dilatation) of vessels and thickness of their walls. This image can allow one to identify abnormal intraluminal and perivascular structures (thrombus or tumour). However, a fresh thrombus may not give reflection as well as blood itself [6].

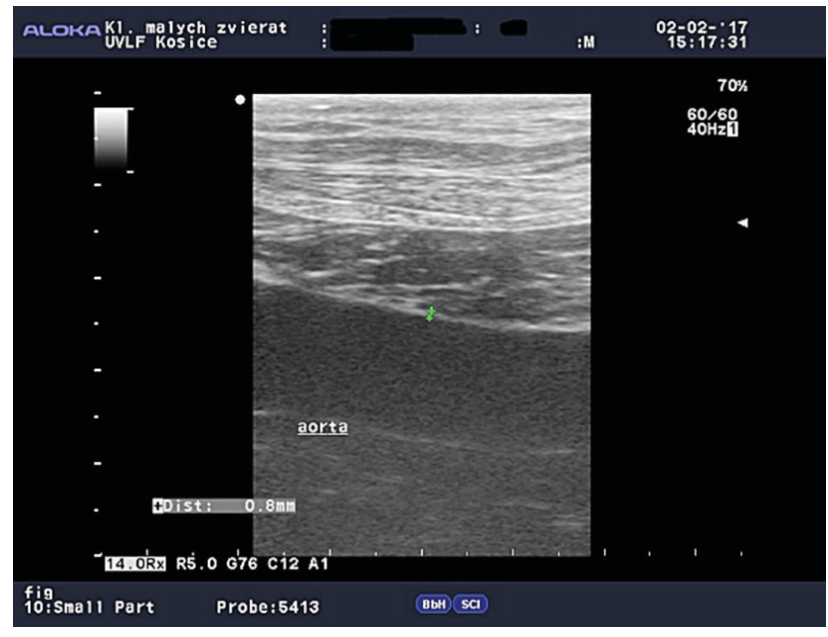

Fig. 2. Sonographic image of a vessel in B-mode. Measurement of wall thickness

Source: Small animal clinic of the UVMP in Košice

\section{HAEMODYNAMICS}

\section{Flow velocity profiles of arteries}

The pulsatile flow in the arteries reaches a maximum velocity during systole and a minimum during diastole. With the systolic ejection of blood into the aorta result- 
1.

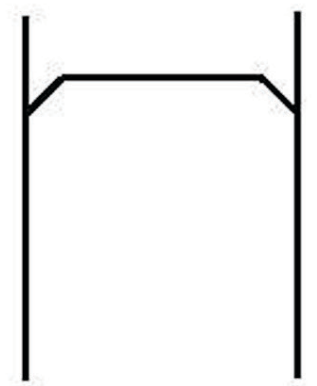

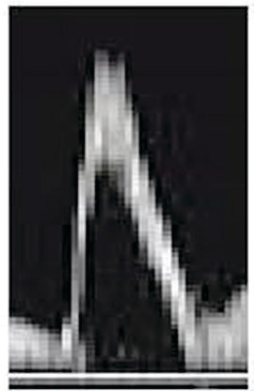

a

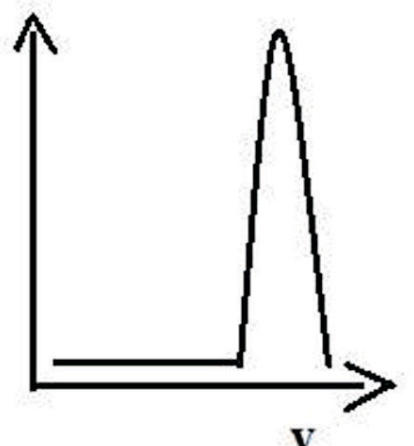

2.

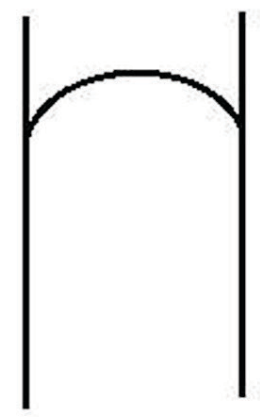

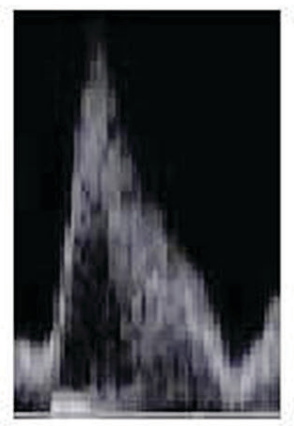

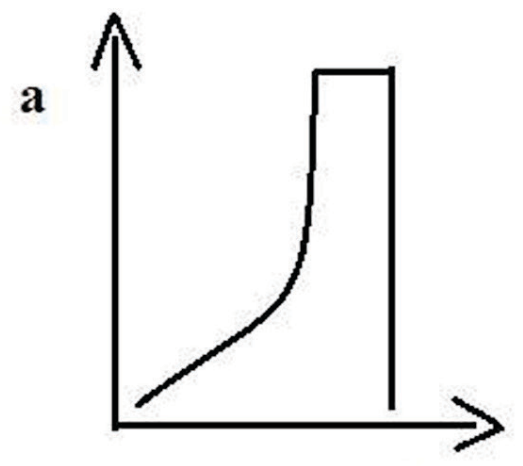

V

3.

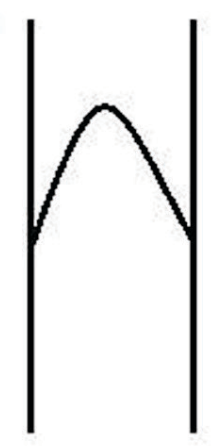

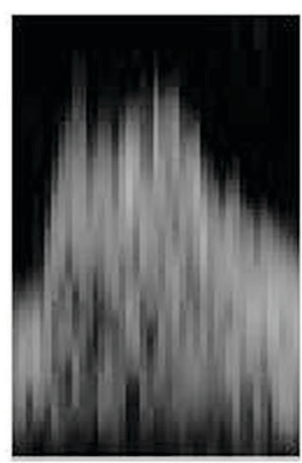

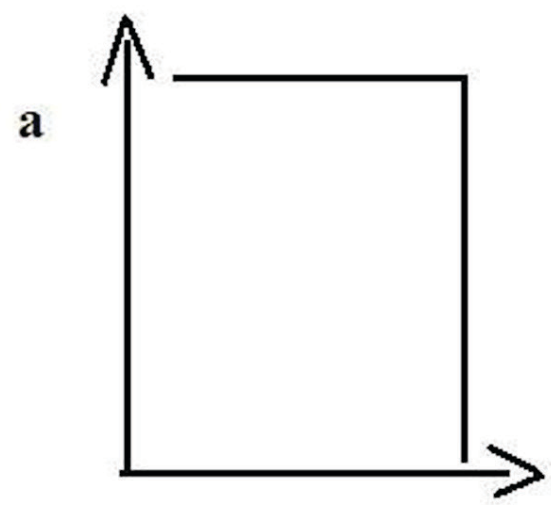

Fig. 3. Flow velocity profiles

1. Cylindrical flow. 2. Blunted parabolic flow. 3. Parabolic flow

Source: Szat mari et al. [14]

ing in pulsatile character of flow, its continuous flow in the periphery is maintained by the elasticity of the walls of the aorta and large vessels (representing blood reservoirs) and resistance of peripheral vessels [3]. The blood flow in most blood vessels is laminar, with the blood moving in thin concentric layers or lamellae. The central layers flow the fastest, whereas frictional forces cause energy loss and slowing of the layers near the vessel walls [6].

The following blood velocity profiles have been described:

1. Cylindrical: In larger vessels, e. g. aorta and its large paired branches, the velocity of the blood is nearly 
the same in the centre of the vessel and near the vessel wall where it is slowed down only moderately (narrow range of frequencies/velocities). As a result, the spectrum is characterised by a thin line in systole that outlines a clear space called a spectral window (empty space between the inner margin of the flow curve and the basal line) [19].

2. Blunted parabolic: This type of flow is basically a combination of the first two types. It occurs in middle-sized arteries (e.g. celiac trunk), the flow is similar to the cylindrical type in the centre of the vessel, however, the flow is more similar to the parabolic type in the peripheral parts of the vessel lumen. (Fig. 3).

3. Parabolic: In smaller arteries (e.g. renal artery) the centrally moving blood has higher velocity compared to the blood close to the vessel wall (wide range of frequencies/velocities),

4. Turbulent: The fourth type of flow velocity profile described in the literature is turbulent. It is observed as bifurcations, curves, stenosis or branches. It is characterised by a wide range of velocities from zero to both the negative and positive maximum velocities [14].

The width of the systolic peak is independent of the flow velocity profile.

1. Cylindrical profile: the overwhelming majority of red blood cells moves with uniform velocity. The spectrum is characterised by a thin line in systole that outlines a space called a spectral window.

2. Blunted parabolic profile: the flow resembles the cylindrical one in the centre of the vessel (red blood cells move with uniform velocity), however, in the peripheral parts of the vessel lumen the flow is more similar to the parabolic flow.

3. Parabolic profile: the centrally moving blood cells have higher velocity in comparison with cells close to the vessel wall, so the distribution of the velocity in the vessel is wide and no spectral window is observed [14].

\section{Doppler waveforms of blood vessels}

The pulsatility of the waveform is related to the vascular impedance downstream to the point of measurement. Each cardiac contraction causes forward blood flow and distension of the vessel. The subsequent diastolic reverse flow is due to blood rebounding up the aorta as the velocity wave is reflected from the high impedance of the peripheral vascular bed of the hind limbs [15]. As the vessel diameter returns to normal, the rebound energy provides the necessary energy to promote continuous flow in diastole $[6,14]$. These types may occur in the body with both low and high flow velocity. Depending upon the organ, tissue or bodily parts, physiological or pathological waveforms may be obtained [3].

The following flow patterns have been described:

1. High resistance flow pattern: High pulsatility and high resistance to flow are indicated by sharp systolic peaks and flow reversal in early diastole (aorta) [14]. It has a three-phase character with high forward flow velocity, low reverse flow at the end of systole and slow forward flow in diastole (the so-called three-peak waveform). It is characterised by a short acceleration time (i.e. time from the onset of flow acceleration in systole up to reaching the maximum velocity) [3]. The high resistance flow pattern is typical of the aorta, limb arteries and external carotids (vessels supplying muscles and skin). It is pathological in the case of increased peripheral resistance in parenchymatous organs (e.g. rejection of renal graft) in places in front of stenosis and vessel occlusions [3].

2. Low resistance flow pattern: Low pulsatility and low resistance to flow are indicated by broad systolic peaks and continuous, high velocity flow in diastole and subsequent permanent flow with gradually decreasing velocity. Acceleration of the flow is slower so a longer time is needed for acceleration and there is no reverse flow at the end of systole. The low resistance flow pattern is characteristic of internal carotids and their branches and arteries supplying the parenchymatous organs with constant need of high flow per minute. It indicates pathology in regions in front of arteriovenous fistulae, in sites with collateral circulation and serious stenosis [3].

3. Intermediate resistance flow pattern: Intermediate pulsatility and intermediate resistance to flow are indicated by sharp systolic peaks (broader than in arteries of high resistance flow pattern) and a forward flow in diastole without reverse flow (e.g. in 
the celiac trunk). The diastolic peak velocity is lower in comparison with the peak systolic velocity than in the low resistance flow pattern [14].

4. Venous flow: Most veins have low grade plasticity and periodicity. The shape of venous flow waveforms depends not only on the velocity of the blood flow, but also on the heart action and respiratory movements. Usually, the flow in the veins is laminar, affected by intrathoracic and intraabdominal pressure conditions (during inspiration and expiration) responsible for phasic changes. Close to the heart, the waveform acquires a three-phase character, with accelerated flow at ventricular systole and at the opening of atrioventricular valves. A short reverse flow appears at atrium systole. For example, v. hepatica and the cranial part of the ventriculo-coronary connection (VCC) have Doppler patterns with strong periodicity because of the effect of the right atrial pressure changes during the cardiac cycle. In the more distant parts of the body (limbs) the venous blood flow is affected mostly by respiration and the work of limb muscles and considerably less by heart action. Inspiration results in acceleration of flow in veins above the diaphragm but in deceleration of flow below the diaphragm. The evaluation of waveforms of veins is mostly based on the description of flow velocity profiles (laminar - turbulent, low - high, three-phase - flat, present - absent). When evaluating venous flow in the limbs of the above mentioned measurements and indices, only measurement of the maximum velocity (Vmax) and flow direction changes (including the length of duration) during various manoeuvers are used [3].

The aim of this study was to determine by Doppler ultrasonography the spectral waveform of vessels of the a. carotis communis, a. femoralis and aa. renales, and to determine their flow velocity profile, resistance flow pattern and values of the RI and PI indices and compare these parameters with previous studies and confirm and extend the database of relevant results.

\section{MATERIALS AND METHODS}

Doppler ultrasonographic examinations of the vessels aa. renales and $a$. femoralis were carried out in a group of
10 clinically healthy dogs weighing $7-26 \mathrm{~kg}$ ( 3 West Highland Terriers of age $5-9$ years, weighing $7-8 \mathrm{~kg}$, and $7 \mathrm{me}-$ dium size mongrels of age $3-9$ years, weighing $15-26 \mathrm{~kg}$ ). The same parameters of the $a$. carotis communis were measured in 6 Miniature Schnauzers of age 2-6 years, weighing $6-8 \mathrm{~kg}$.

After arrival at the clinic, the prospective dogs were allowed to acclimatize and get used to the new environment for 5-10 minutes. Those that reacted wildly to stress factors (manipulation of the animals, unknown environment, length of the examination) were excluded from the study.

The selected dogs were examined without sedation after fasting for 12 hours, using an ultrasonographic apparatus Aloka Profound Alpha 6, equipped with linear (5$16 \mathrm{MHz})$ and microconvex $(5-10 \mathrm{MHz})$ probes.

\section{RESULTS}

The Doppler ultrasonographic examinations of the a. femoralis revealed a high resistance flow pattern and a cylindrical flow velocity profile with a spectral window. The systolic peak was followed by a retrograde flow in early diastole and then again by forward flow and lower reverse flow and ended by a forward flow (Fig. 4). The spectral pattern of the $a$. femoralis showed a waveform corresponding to the respiratory movements, heart action or transfer of pulsatility from the adjacent artery (Fig. 5).

The Doppler spectral pattern of the aa. renales was characteristic of a low resistance flow pattern and parabolic flow velocity profile. An early systolic peak (ESP), typical of this artery, can be seen in Fig. 6. The impedance indices in the medium size breeds were as follows: $\mathrm{RI}=0.632 \pm 0.05$; $\mathrm{PI}=1.153 \pm 0.02$. In the medium size mongrels these indices reached: $\mathrm{RI}=0.688 \pm 0.08 ; \mathrm{PI}=1.341 \pm 0.08$. In the West Highland Terriers, the RI index was $0.734 \pm 0.03$ and PI 1 . $605 \pm 0.03$. The mean values of indices for all size categories were the following: $\mathrm{RI}=0.684 \pm 0.05 ; \mathrm{PI}=1.366 \pm 0.04$.

The spectral pattern of $a$. carotis communis was specific. The systolic peak was broader with small, not very evident spectral window (blunted parabolic flow velocity profile). The systolic peak was not followed by a retrograde wave in early diastole (intermediate resistance flow pattern) but by continuous forward flow. This involved the so-called intermediate resistance waveform (combination of two arteries, a. carotis interna and arteria carotis externa) (Fig. 7). 


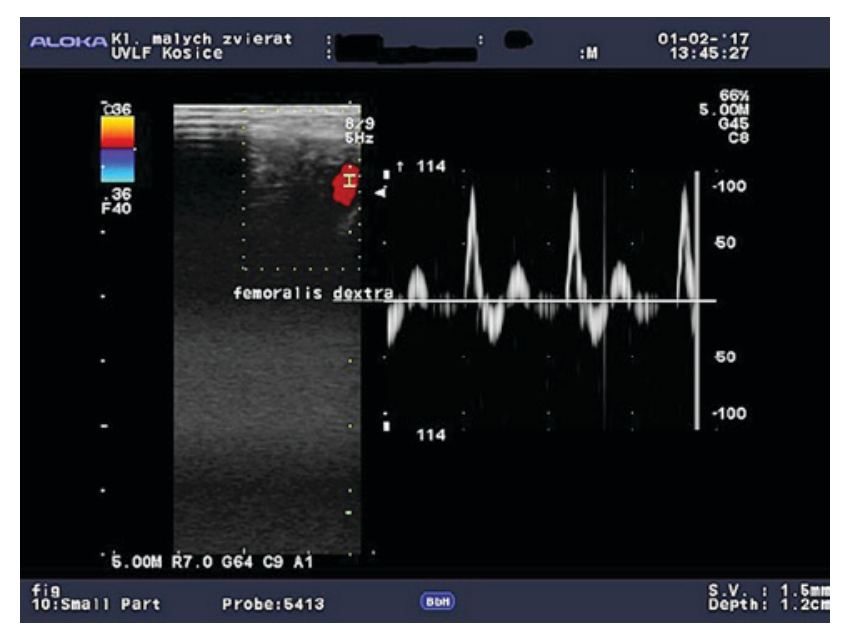

Fig. 4. Spectral Doppler pattern of a. femoralis dextra Source: Small animal clinic of the UVMP in Košice

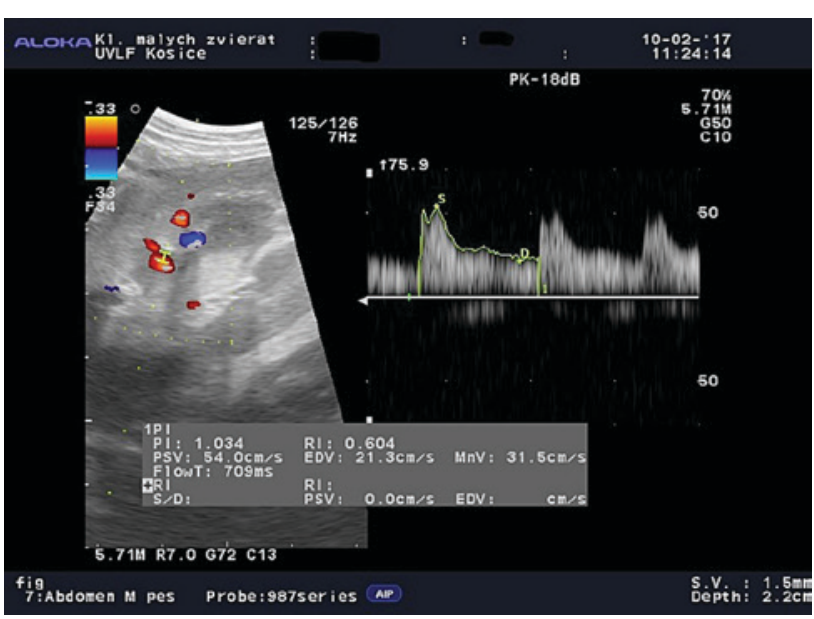

Fig. 6. Spectral Doppler pattern of aa. renales Source: Small animal clinic of the UVMP in Košice

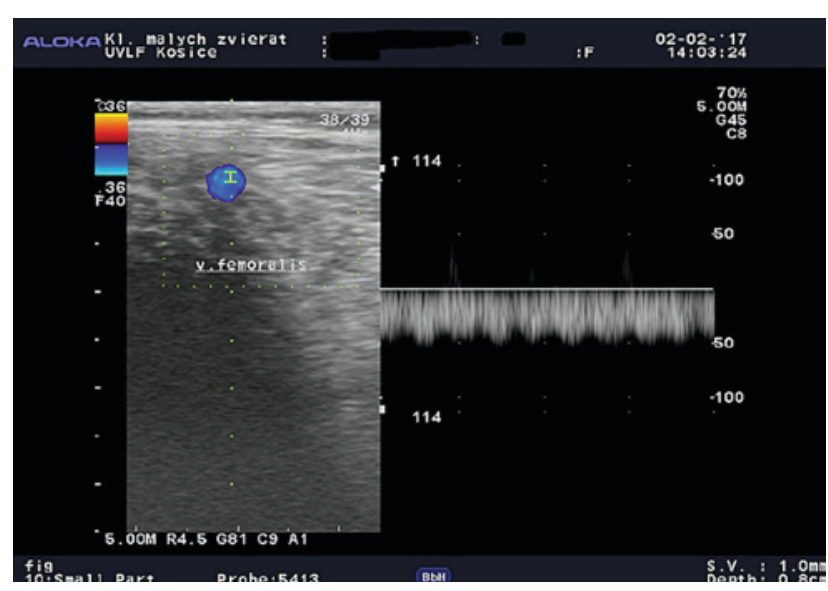

Fig. 5. Spectral Doppler pattern of $v$. femoralis Source: Small animal clinic of the UVMP in Košice

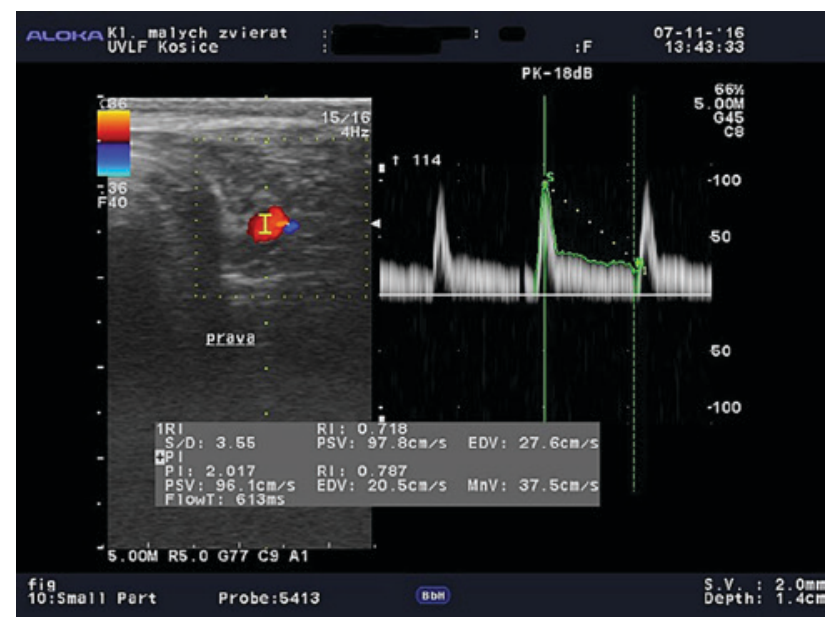

Fig. 7. Spectral Doppler pattern of a. carotis communis Source: Small animal clinic of the UVMP in Košice

studies have been published dealing with the physiological values of the RI and the PI of individual vessels. In our study we investigated small and medium size dogs.

The $a$. femoralis is a vessel with a high resistance flow pattern and cylindrical flow velocity profile. Access to this vessel and its examination are not simple. There was observed a sharp systolic peak with a clear spectral window. Its flow velocity distribution was narrow and the systolic peak was followed by reverse flow in early diastole and then again by forward flow. The mean peak systolic velocity (PSV) in this artery in the study by Lee et al. [7] was $110 \pm 17 \mathrm{~cm} . \mathrm{s}^{-1}$. The mean early diastolic velocity (EDV) was $11 \pm 5 \mathrm{~cm} \cdot \mathrm{s}^{-1}$ and end diastolic velocity (EnDV) reached $22 \pm 7 \mathrm{~cm} . \mathrm{s}^{-1}$ [7]. The differences in the results of the left and colour and power Doppler mapping. In cardiology, the spectral Doppler examination is used most commonly. Few 
right $a$. femoralis were insignificant. The examination of the a. femoralis was indicated because of the manifestation of weakness of hind limbs or a weak femoral pulse. Its typical three-phase waveform was subject to rapid changes in pathologies such as stenosis, thrombus obstruction, tumour, arteriovenous malformation (AVM) and other abnormalities. Similar pathological signs were described in systemic arterial dirofilarasis [17].

The aa. renales is a vessel with a low resistance flow pattern and parabolic flow velocity profile. The mean values of the impedance indices in this vessel reached $0.684 \pm 0.05$ for the RI and $1.366 \pm 0.04$ for the PI without significant difference between right and left limbs. In the medium breeds, the RI reached $0.632 \pm 0.05$ and the PI $1.153 \pm 0.02$ and in the medium size mongrels, the RI was equal to $0.688 \pm 0.08$ and the PI 1.341 \pm 0.08 . In the West Highland Terriers, we measured $0.734 \pm 0.03$ for the RI and $1.605 \pm 0.03$ for the PI.

A urinary obstruction or vasoconstriction can increase the resistance of the renal vessels. In the case of the increased vascular resistance, the diastolic flow of blood is decreased more than the systolic one. The relatively higher decrease in the end diastolic velocity compared to the systolic velocity results in increased values of the RI and the PI. Thus, it is necessary to determine the upper limits for the RI and the PI in order to identify abnormally increased vascular resistance [9].

According to Mattoon and Nyland [8], every value of the RI higher than 0.7 is considered abnormal. Such findings are non-specific and occur in a number of disorders such as acute renal disease, acute tubular necrosis and renal obstruction [8]. The authors conducted a study on 27 dogs without sedation and the upper limits were 0.72 for the RI and 1.52 for the PI. They examined the vessels by the power Doppler technique. The differences in the measured values between the right and left kidneys were insignificant [9]. In the study by Och oa et al. [10], the limit for the RI was higher and the results had to exceed $0.75-0.8$ to be considered abnormal.

The examination of the RI is important in case of acute obstructions. An increased RI was recorded 24 hours following the acute unilateral obstruction in dogs, however, due to the high number of false positive and false negative cases the reliability of the RI examination was limited [16]. Despite that, an increased RI and difference in the RI between both kidneys higher than 0.1 can support a detection of an acute unilateral obstruction in dogs [8].
Bude et al. [4] presented in their study the values of renal RI, which remained unchanged in kidneys without obstruction and increased in kidneys with obstruction following administration of furosemide. They described the effect of furosemide in combination with salineF1/1 on the renal RI. The authors evaluated the renal RI in children and juveniles aged 6 to 18 years before and after i.v. administration of furosemide, in combination with saline (F1/1). The $\mathrm{RI}$ index was increased in the obstructed kidney.

The administration of an infusion of F1/1 solution in combination with furosemide (diuretics) to dogs resulted in an increased RI in the kidney with experimentally induced obstruction and a decrease in the RI in the nonobstructed kidney $[5,11,18]$. The difference was primarily ascribed to the decrease in the RI index in the kidney free of obstruction. This "furosemide challenge" test technique is helpful in the detection of post-transplacental obstruction in dogs [8]. Our study presented physiological values in clinically healthy individuals of medium size dogs. The only exception was one dog; a West Highland Terrier with a RI value reaching $0.734 \pm 0.03$.

The study on dogs with leishmaniasis and various stages of renal damage showed that the RI index can serve as a reliable indicator of progressivity of the problem with high sensitivity but small specificity. All investigated animals exhibited an increased RI value, which suggested real advanced damage and proteinuria $[1,2]$.

The characteristics of $a$. carotis communis reflect differences in its branches, $a$. carotis interna and a. carotis externa. In the group of dogs observed in our study, the examinations showed medium pulsatility (atypical high resistance waveform with absence of reverse flow) and a blunted cylindrical flow velocity profile. Its waveform differs also in individual size categories of dogs. The mean values of a. carotis communis measured in the Miniature Schnauzers were as follows: left side $\mathrm{PI}=1.824$ and $\mathrm{RI}=0.742$; right side $\mathrm{PI}=1.891$ and $\mathrm{RI}=0.746$. In comparison, the $\mathrm{RI}$ and the PI in the left carotid of West Highland Terriers reached 0.715 and 1.677, respectively. In the right carotid of this breed, the RI and the PI were 0.788 and PI 1.912 respectively. In the Miniature Schnauzers the mean PSV was $71.7 \mathrm{~cm} . \mathrm{s}^{-1}$ and EDV $17.8 \mathrm{~cm} \cdot \mathrm{s}^{-1}$.

A pioneer evaluation of $a$. carotis communis was presented by Svicero et al. [13] who described their ultrasonography results in a Labrador breed. The aim of their study was to determine the physiological values of PSV, 
EDV and RI. These authors did not observe significant differences between the right and the left vessels, the PSV reached $75.8 \pm 16 \mathrm{~cm} . \mathrm{s}^{-1}$, EDV $12.2 \pm 4 \mathrm{~cm} . \mathrm{s}^{-1}$, diameter of a. carotis communis was $0.54 \pm 0.063 \mathrm{~cm}$ and the RI was $0.83 \pm 0.07$. Lee et al. [7] described in their study the physiological parameters of blood flow velocity in a. carotis communis and the PI values of $a$. basilaris. The mean PSV of $a$. basilaris and a. carotis communis were $72 \pm 19$ and $115 \pm 17$, respectively. The $a$. carotis communis were $25 \pm 11$ and $39 \pm 7$, respectively. The PI of the basillary artery was $1.37 \pm 0.13$. The mean systolic and diastolic blood pressures were $137 \pm 13$ and $78 \pm 15 \mathrm{mmHg}$, respectively.

The mean values of the RI and the PI indices of $a$. carotis communis and aa. renales obtained in our study agreed with the values reported by the above authors. Our results extend the database of the physiological values of these vessels.

\section{CONCLUSIONS}

The Doppler ultrasonographic examination of the a. femoralis is unquestionably a simple, excellent and noninvasive method of examination of this vessel that should be used as one of the examination methods when various pathological states of hind limbs are suspected.

The mean values of the RI and PI indices of aa. renales were: RI $0.684 \pm 0.05$; and PI $1.366 \pm 0.04$. These results are comparable with those obtained by other researchers. The RI results obtained in our study were below 0.7 . We consider this a physiological limit. The exception was the West Highland terrier breed with a PI equal to 0.734 . However, we examined only a very small sample of this breed.

The use of the RI as a marker of renal damage still seems controversial and obtaining the waveforms of good quality appears time and technique demanding.

The mean values of the indices of $a$. carotis communis for the Miniature Schnauzers were: left side PI $=1.824$ and $\mathrm{RI}=0.742$; right side $\mathrm{PI}=1.891$ and $\mathrm{RI}=0.746$. The mean value of PSV was $71.7 \mathrm{~cm} . \mathrm{s}^{-1}$ and of EDV $17.8 \mathrm{~cm} . \mathrm{s}^{-1}$. Our results corresponded to those reported by other researchers. These physiological values of the Miniature Schnauzer breed can serve for comparison in the study of pathological changes that correlate with the pathology of neurological disorders with an ischemic basis, or at thromboembolic states, atherosclerosis, arterial stenosis or tumours in the cervical region.
Our results obtained for the $a$. carotis communis in the Miniature Schnauzer can become a breed standard of characteristics of flow in this vessel.

\section{ACKNOWLEDGEMENT}

The study was supported by the project VEGA No. 1/0225/15.

\section{REFERENCES}

1. Baltazar, P. I., Da Silva Moura, L. S., Pessoa, G. T., Rodrigues, R.P.S., Sanches, M.P., Diniz, A.N. et al., 2016: Comparative B-mode and Doppler renal ultrasonography with histopathological findings in dogs positive for canine visceral leishmaniasis. Microsc. Res. Tech., 79, 637-645.

2. Braga, J.F. V., Alves, F. R., 2016: Comparative B-mode and Doppler renal ultrasonography with histopathological findings in dogs positive for canine visceral leishmaniasis. Microsc. Res. Tech., 79, 637-645.

3. Beňačka, J., Tvrdík, E., 2013: Doppler basis (In Slovak). In Proceedings of the 9th Congress of Slovak Sonography, Slovakia, Pieštany, 5.

4. Bude, R. O., DiPietro, M.A., Platt, J.F., Rubin, J.M., 1994: Effect of furosemide and intravenous normal saline fluid load upon the renal resistive index in nonobstructed kidneys in children. J. Urol., 151, 438-41.

5. Choi, H., Won, S., Chung, W., Lee, K., Chang, D., Lee, H. et al., 2003: Effect of intravenous mannitol upon the resistive index in complete unilateral renal obstruction in dogs. J. Vet. Intern. Med., 17, 158-62.

6. Finn-Bodner, S., Hudson, J. A., 1988: Abdominal vascular sonography. In Penninck, G. (Ed.): The Veterinary Clinics of North America. Small Animal Practice. Ultrasonography. Philadelphia, W. B. Saunders, 28, 887-942.

7. Lee, K., Choi, M., Yoon, J., Jung, J., 2004: Spectral waveform analysis of major arteries in conscious dogs by Doppler ultrasonography. Veterinary Radiology and Ultrasound, 45, 166-171.

8. Mattoon, J. S., Nyland, T. G., 2015: Small Animal Diagnostic Ultrasound, 3th edn., Missouri, Elsevier, 32-47, 125-127, 431-435, 581-584.

9. Novellas, R., Espada Y., de Gopegui, R.R., 2007: Doppler ultrasonographic estimation of renal and ocular resistive and 
pulsatility indices in normal dogs and cats. Vet. Radiol. Ultrasound, 48, 69-73.

10. Ochoa, PG., Lacasta, D., Sosa I., Gascon, M., Ramos, J. J., Ferrer, L.M., 2011: Fundamentals and Applications of Abdominal Doppler. Ultrasound Imaging - Medical Applications. Chapter 14, Minin, I. V., Minin, O. V. (Eds.), published by InTech under CC BY-NC-SA 3.0 license, 342 pp.

11. Shokeir, A.A, Nijman, R.J., el Azab, M., Proovost A.P., 1997: Partial ureteral obstruction: Effect of intravenous normal saline and furosemide upon the renal resistive index. J. Urol., 157, 1074-7.

12. Spaulding, K. A., 1997: A review of sonographic identification of abdominal blood vessels and juxtavascular organs. Vet. Radiol. Ultrasound, 38, 4-23.

13. Svicero, D. J., Doiche, D. P., Mamprim, M. J., Heckler, M. C., Amorim, R. M., 2013: Ultrasound evaluation of common carotid artery blood glow in the Labrador retriever, BMC Veterinary Research, 9,195. http://www.biomedcentral.com/1746$61 / 9 / 195$.

14. Szatmari, V., Sotonyi, P., Voros, K., 2001: Normal duplex Doppler waveforms of major abdominal blood vessels in dogs: A review. Vet. Radiol. Ultrasound, 42, 93-107.
15. Taylor, K. J., Burns, P. N., Woodcock, J. P., Wells, P. N., 1985: Blood flow in deep abdominal and pelvic vessels: ultrasonic pulsed-Doppler analysis. Radiology, 154, 487-493.

16. Ulrich, J.C., York, J.P., Koff, S.A., 1995: The renal vascular response to acutely elevated intrapelvic pressure: resistive index measurements in experimental urinary obstruction. J. Urol., 154, 1202-1204.

17. Upchurch, D.A., Ogden, D.M., Baker, D.G., 2015: Bilateral femoral arterial dirofilariasis caused by Dirofilaria immitis in a dog. Veterinary Record Case Reports, 3: e000184. doi: 10.1136/vetreccr-2015-000184.

18. Yokoyama, H., Tsuji, Y., 2002: Diuretic Doppler ultrasonography in chronic unilateral partial ureteric obstruction in dogs. BJU Int., 90, 100-104.

19. Zwiebel, W. J., Fruechte, D., 1992: Basics of abdominal and pelvic duplex, instrumentation, anatomy and vascular Doppler signatures. Semin. Ultrasound CT MR, 13, 3-21.

Received August 24, 2017

Accepted October 6, 2017 